\title{
Philosophy of Medincine
}

On the Brink of Disaster

Vulnerability to Mental Disorders

\author{
Valentina Petrolini ${ }^{1}$ \\ 1 Department of Linguistics and Basque Studies, Micaela Portilla Research Center, University of the Basque \\ Country (UPV/EHU), Vitoria-Gasteiz, Araba, Spain. Email: valentina.petrolini@ehu.eus
}

\begin{abstract}
The notions of at-risk and subthreshold conditions are increasingly discussed in psychiatry to describe mild, brief, or otherwise atypical syndromes that fail to meet the criteria for clinical relevance. However, the concept of vulnerability is still underexplored in philosophy of psychiatry. This article discusses psychiatric vulnerability to clarify some conceptual issues about the various factors contributing to vulnerability, the notions of risk and protection, and the idea that there are multiple ways of crossing the threshold to clinical relevance. My goal is to lay the groundwork for a finer-grained discussion on psychiatric vulnerability that reflects the complex nature of mental conditions and illustrates the kind of thinking needed in clinical practice.
\end{abstract}

\section{Introduction}

The notion of vulnerability is intuitively connected to the idea of being exposed, weakened, or otherwise in need of help or protection. Everyday uses of the word vulnerability convey this idea in different ways: some people are considered vulnerable because of their physiological status (for example, the elderly), or because of psychological characteristics (for example, low self-confidence). Several aspects of vulnerability have been explored by researchers in medical ethics and feminist philosophy. In these works, vulnerability has been mostly characterized in terms of increased susceptibility to harm, weakness, dependency, or powerlessness (Boldt 2019; Bluhm 2012). Relatedly, vulnerable people-or groups-are described in terms of structural or epistemic injustice, as those exhibiting a greater likelihood of being denied adequate satisfaction of legitimate claims concerning, for example, physical integrity, autonomy, freedom, and so on (Clark and Preto 2018; Tavaglione et al. 2015). Erinn Gilson (2011) offers a different account that emphasizes the ambivalent nature of vulnerability. Despite the fact that being vulnerable implies negative consequences (such as diminished autonomy or agency), it also constitutes a necessary condition for openness-as the ability to be positively or negatively affected by the world and others. In this sense, vulnerability may be seen as an integral part of being human, and as a potential driver for positive change.

Within medical practice, the notion of vulnerability has a different connotation and a more specific use, which is the main focus of this article. Vulnerability in medical settings

\section{$(\mathrm{cc}) \mathrm{EY}$}

Articles in this journal are licensed under a Creative Commons Attribution 4.o United States License.

(C) The Author(s). Published by the University Library System, University of Pittsburgh. ISSN 2692-3963 | Date Received 28-08-2020 | Date Accepted 15-09-2021 | Date First Published 01-10-2021 Philosophy of Medicine | DOI 10.5195/pom.2021.10 | Volume 2 | Issue 2 | pp.1-21 
is usually connected to the idea of relative risk; that is, to the fact that some individuals or groups are more likely to encounter harm than others. ${ }^{1}$ A reliable and effective identification of such individuals is important in medicine for reasons ranging from the allocation of resources, prevention, and intervention on the factors thought to increase or diminish risk. A more precise characterization of vulnerable individuals and groups may help clinicians in the diagnostic process and in clinical case formulations, resulting in a more individualized approach to prevention and treatment (Van Os 2013). However, the notion of vulnerability as relative risk also poses methodological and theoretical challenges. From a methodological perspective, it is unclear how such a greater likelihood of being exposed to harm may be effectively measured, or how clinicians may reliably identify groups or individuals that would count as vulnerable (Travaglia and Robertson 2013). Theoretically speaking, the connection between vulnerability and relative risk may be interpreted in different ways, depending on the view of possibility that is adopted (more on this below).

In the following section I take a closer look at the conceptual issues surrounding vulnerability (section 2.1), and then I briefly distinguish between different contributing factors that determine an individual's overall degree of vulnerability (section 2.2). In sections 3 and 4 I focus on the key notions of risk and protection, respectively, and show how they apply to some exemplar cases. Specifically, I substantiate the idea that there are multiple paths from vulnerability to pathology; that is, multiple ways of crossing the threshold to clinical relevance.

\section{Vulnerability in Psychiatry}

\subsection{Conceptual Issues}

Vulnerability is connected to possibility by virtue of being a modal property that concerns what would or could happen. As fragility may be seen as the propensity to break, vulnerability may be seen as the propensity to be exposed to harm. If we embrace a broad notion of possibility-that is, what may statistically occur-vulnerability may be simply characterized in terms of the increased likelihood that a given individual or group would encounter harm. Notably, this notion remains agnostic with respect to the connection between the individual's (or group's) features and the harm encountered. For instance, one may claim that people living in urban settings are more likely to develop schizophrenia without thereby committing to the idea that urban living is causally-or otherwise-connected with the development of this condition. However, if we adopt a more restrictive view of possibility-such as the one defended by Barbara Vetter (2014)vulnerability could be described in terms of sets of dispositions that impinge on relevant properties or features displayed by individuals or groups. In this reading, vulnerability should be seen as susceptibility to harm, as the idea that subjects can encounter harm due to particular relevant properties or features (Travaglia and Robertson 2013). So, in the example of schizophrenia above, this more restrictive view of vulnerability would urge us to take into account relevant-and preferably causal-connections between urban living and the development of schizophrenic symptoms.

1 This notion of relative risk is broader than the one used in epidemiological and cohort studies, which is commonly referred to as risk ratio. In those contexts, the notion is normally used to measure the difference between exposure and outcome in different groups-for example, to compare the risk of an adverse outcome when receiving medical treatment versus no treatment (or placebo). 
This distinction is important because it allows us to weed out potentially spurious correlations between vulnerability and risk. Indeed, there may be situations in which someone exhibits a de facto higher relative risk due to factors that turn out to be not causally connected with the relevant condition. For instance, we may discover that the connection between urban living and schizophrenia was actually dependent on yet another factor (for example, social isolation) which exhibits a causal relation with the development of the condition. In this case, the initial correlation would still be included in the broader definition of vulnerability as relative risk, but it would not be particularly informative for clinical purposes. Although it would initially help clinicians to isolate a subgroup of individuals at higher risk of developing schizophrenia (those living in an urban setting), it would fail to pick out the clinically relevant subgroup-people who suffer from social isolation.

By contrast, the more restrictive view of possibility gets us closer-at least ideally-to the notion of vulnerability that I want to explore in this article. In this view, an individual or group can be said to be more vulnerable to a given condition if they exhibit a number of (dispositional) features that are causally connected with the development or exacerbation of clinically relevant symptoms. ${ }^{2}$ In this sense, a mere increase in relative risk may be insufficient for an individual or group to qualify as more vulnerable. In fact, many instances of increased risk are not going to be relevant with respect to the potential development of a given condition. As a consequence, psychiatric vulnerability should be seen as a specific subset of increased relative risk, one including factors or features that are causally connected with the relevant condition. ${ }^{3}$

Yet, identifying individuals or groups at higher risk of developing mental conditions is far from straightforward. For instance, in the study of psychosis, several labels have been introduced to refer to subjects at high risk of developing clinically relevant conditions, such as "at risk mental state" (ARMS), "ultra high risk" (UHR), and "clinical high risk" (CHR) (Fusar-Poli et al. 2016). These high-risk states are usually characterized in terms of attenuated psychotic symptoms-for example, brief, limited, or intermittent psychotic episodes-or through the identification of subtle cognitive, emotional, or perceptual disturbances (Fusar-Poli et al. 2013). Despite their success in identifying segments of atrisk populations (McGorry et al. 2006; Broome et al. 2005), ARMS and related constructs appear to lack specificity when it comes to predicting the transition from vulnerability to pathology. An important issue concerns the rate of "false positives," individuals who are identified as being at risk but then fail to develop a psychotic disorder in the following two to three years (Ajnakina, David, and Murray 2019). To sidestep this issue, some researchers have proposed to design prevention programs by taking into account more general risk factors associated with mental health (for example, childhood abuse, migration, cannabis use, and so on-see Murray, David, and Ajnakina 2021; Modinos et al.

\footnotetext{
2 This also implies that vulnerability is gradable, and that people or groups may be more or less vulnerable depending on a host of factors. Drawing on Vetter's example on irascibility, we may say that A is more vulnerable than B if A can be harmed more easily and in a wider variety of circumstances (Vetter 2014, 142). 3 Given the complexity surrounding etiological factors for mental conditions, this view of vulnerability currently works more as a regulative ideal. However, establishing a causal taxonomy for mental conditions as significant departures from normal functioning is a crucial enterprise for psychiatry as a branch of medicine (Murphy 2006). Even if our causal knowledge is far from complete, we can start by discriminating among potentially relevant factors that may give us helpful indications on how to circumscribe vulnerable individuals or groups. The focus on causal connections should not obscure the fact that some correlations are clinically useful even in the absence of causal underpinnings. For instance, in somatic medicine we may understand that a disease is spread through the exchange of bodily fluids, and thus design public health interventions accordingly, even before we can reliably identify the specific viral agent.
} 
2014). A preliminary distinction that should be drawn is thus the one between ARMS and related notions, on the one hand, and subthreshold extended phenotypes, on the other (Van Os 2013). While the former refer to individuals who seek clinical help at mental health services, the latter refer to members of the general population who do not seek help despite exhibiting elements of vulnerability. These people are also at times characterized as fragile (Kottow 2003) as they exhibit a more generalized and symptom-unspecific exposure to risk than people detected by ARMS or similar programs.

This further suggests that the possibility of drawing a boundary between vulnerable and disordered conditions hinges on a more fundamental distinction between health and pathology. This conceptual challenge has accompanied psychiatry for decades, especially following the critiques raised in the 1960s about the risk of pathologizing "problems of living" (Szasz 1960; Foucault 1961). What makes a mental condition serious enough to warrant clinical intervention? Is it possible to distinguish between disorder and normal suffering? Diagnostic manuals acknowledge the complexity surrounding these questions and tend to characterize disorders pragmatically, for instance as "clinically recognizable set(s) of symptoms or behavior associated in most cases with distress and with interference with personal functions" (World Health Organization 1992, ICD-10; see also Powell and Scarffe 2019 and Kingma 2017 for a review of philosophical debates on the notion of disorder).

In this article I adopt a similarly pragmatic approach. In this view, the question of what counts as disordered may be connected-although not fully reduced-to the issue of what counts as clinically relevant. This notion encompasses the range of conditions that people "bring to the clinic" and refers to the way in which severity, distress, and harm are expressed and observed in the clinical encounter (Bolton 2008). From the clinician's perspective, pathology is primarily assessed on the basis of holistic considerations about the individual's relevant goals, contextual circumstances, and the existence of a perceived problem, followed by a search for its causes according to the current diagnostic and medical guidelines (Nordenfelt 2007). A healthy-or not clinically relevant-situation is therefore one in which an individual does not seek psychological help, and nor do the people surrounding her believe she would benefit from treatment. By contrast, a pathological-or clinically relevant-situation is one in which an individual perceives a problem in a broad sense, including bodily or psychological pain, the impossibility of doing what they would normally do, and other forms of suffering (Fulford 1989). This implies that the diagnostic boundaries between health and pathology are often determined on a case-by-case basis by taking into account indicators of severity concerning the number, intensity, pervasiveness, and duration of symptoms, as well as the degree of functional impairment (Maj 2011). In such a framework, someone qualifies as vulnerable if they are on the verge of crossing the threshold to a clinically relevant condition-that is, of seeking or needing clinical help. ${ }^{4}$

A further conceptual clarification is in order. The focus on vulnerability and subthreshold conditions intuitively goes hand in hand with a dimensional view of

\footnotetext{
4 I acknowledge that such an approach presents several limitations: for instance, it may raise issues of epistemic authority and injustice (Drożdżowicz 2021; Tate 2019) and it assumes a degree of cross-cultural agreement that is far from being established within psychiatric practice (Lewis-Fernández and Kirmayer 2019). Generally speaking, it runs the risk of enforcing a coercive use of psychiatry in fixing the boundaries around normality and pathology (see Bolton 2008, ch. 5 for a review). However, this rough-and-ready definition allows us to see mental disorders as a form of suffering that may find partial solutions through clinical intervention. In this sense, this approach may still be used as a starting point to unpick the notion of vulnerability starting from existing practices.
} 
psychopathology, in which mental disorders are seen as continuous with extended phenotypes (Van Os et al. 2009). Yet, a pragmatic approach-such as the one outlined above-implies the need to determine whether and when a threshold to clinical relevance has been crossed. In other words, the very idea of vulnerability indicates an increased likelihood to develop or be affected by a particular condition, thereby making it necessary to draw a boundary between clinically relevant and nonclinically relevant states. In this sense, a fully dimensional approach fails to meet a crucial requirement, as it risks making the distinction between health and pathology entirely conventional.

In what follows I outline a threshold approach, in which the shifting point from vulnerability to clinical relevance hinges on a variety of considerations. By introducing the idea of contributing factors, I stress the fact that the transition from vulnerability to pathology involves the clustering of different elements (psychological, environmental, and so on). By discussing different paths, I focus on the idea that the transition to pathology often hinges on an increase in intensity, frequency, or duration of particular symptoms, or on the decrease of protective resources. Although the accumulation of these potentially problematic factors is quantitative in nature, crossing the threshold to pathology marks the entrance into a meaningfully different state. From a clinical perspective, vulnerable and pathological cases warrant different interventions, require different degrees of concern and monitoring, and determine differential access to treatment. The threshold view I outline here thus contributes to partially relaxing the tension between dimensional and categorical approaches (see Serpico 2020 for a similar proposal). It does so by acknowledging that the focus on quantitative differences offers a conceptually finergrained view, while maintaining that qualitative distinctions are clinically and practically important.

\subsection{Unpacking Vulnerability: Contributing Factors and Paths}

In this subsection I distinguish between factors contributing to vulnerability and paths from vulnerability to clinically relevant states. The distinction is particularly important because it allows us to pry apart two aspects of risk that often interact with each other. On the one hand, each individual (or group) occupies a certain position along the continuum between clinical and nonclinical. On the other hand, different factors or forces affect whether and how an individual (or group) along such a continuum is going to develop a clinically relevant manifestation (Johns and Van Os 2001). My proposal here is methodological: more than introducing new heuristic tools to be picked up by clinicians, I unpack and illustrate the kind of thinking that is needed to assess vulnerability in a psychiatric setting. This is particularly complex, given that most conditions tend to be heterogeneous in their presentation, and are usually regarded as multifactorial in their causation (Allsop et al. 2019).

Contributing factors are those affecting an individual's (or group's) degree of relative risk: in other words, these factors determine what makes an individual more susceptible to harm than others. Following the distinction outlined above between different views of possibility, it is important to start by isolating such features or potential determinants and then proceed by dissecting the ones that are relevant-that is, causally connected with the condition at hand. A first step consists in acknowledging that pathogenic factors may be psychological (for example, personality traits) or environmental in nature (for example, traumatic events). We may thus think about contributing factors as features that a person has (for example, neuroticism) or as experiences that she has undergone (for example, childhood abuse) that place her at higher risk in comparison with another individual who 
lacks the relevant features or did not undergo sufficiently similar experiences. Such a distinction has been historically captured by Sigmund Freud's notions of dispositions and experiences: the former are pathogenic determinants "which a person brings along with him into his life," while the latter are "those which life brings to him" $(1913,317)$. In what follows I refer to dispositions as individual factors and to experiences as environmental factors.

Researchers working within the stress-vulnerability model of psychopathology (Ormel and Neeleman 2001; Zubin and Spring 1977) have already stressed that different contributing factors interact with mediating variables and give rise to clinically relevant manifestations. For example, someone who exhibits a tendency toward rumination and has experienced childhood abuse would be more likely to cross a clinically relevant threshold if confronted with further negative events in their life. Crucially, different factors are bound to interact with one another and bring about a complex constellation of vulnerability profiles. People may be differently equipped to deal with environmental stressors because of individual differences, such as personality traits (for example, neuroticism) or affective makeup (for example, a tendency toward anxiety or rumination) (Van den Bergh et al. 2020; Nolen-Hoeksema, Wisco, and Lyubomirsky 2008). Equally, environmental circumstances may increase one's exposure to stressors and therefore affect individual-level factors, to the point that some events-for example, unemployment or childbirth-have come to be known as "turning points" because of their impact on mental health (Turner and Lloyd 1999).

It is also crucial to acknowledge that stress exposure is not uniformly distributed across groups, and that people of different genders, ethnicity, and socioeconomic status are differently affected by stressful life events (Turner and Avison 2003). While some forms of vulnerability overwhelmingly affect individuals, populations may also be seen as vulnerable due to differential exposure to stressors. For instance, people who belong to visible minorities are more likely to be discriminated against in their everyday life than people who belong to the ethnic majority. Similarly, recent mothers may be more prone to developing depressive symptoms due to their heightened exposure to social pressures, and cognitive and emotional loads. In these cases, higher exposure to environmental stressors significantly affects mental health. While migrant groups have been repeatedly found to be at higher risk of developing psychosis (Misra et al. 2021; Fearon, Kirkbride, and Morgan 2006; Veling et al. 2008), postpartum depression has also been linked to social factors such as employment status and degree of family support (Fairbrother and Woody 2008). Members of socially disadvantaged groups who also exhibit individual-level aspects of vulnerability are thus more likely to develop clinically relevant manifestations. 5

Paths from vulnerability to pathology may be characterized as different ways of crossing the clinically relevant threshold. Given an existing degree of vulnerability, determined by complex interaction among the factors outlined above, a person or group may cross the threshold to clinical relevance in multiple ways. For instance, someone may exhibit mild or attenuated symptoms and then experience a sudden increase in severity. This would be the case of a person experiencing auditory verbal hallucinations (AVHs)

${ }^{5}$ Exploring the route from vulnerability to pathology in the case of populations is beyond the scope of this article, especially considering that the interaction between individual and environmental factors is unlikely to be linear and merely additive. Several issues would be interesting to explore, such as the action exercised by repeated experiences of exclusion and discrimination (Cantor-Graee and Selten 2005; Bourque, Van der Ven, and Malla 2011; Berg et al. 2011), the reasons why specific conditions are more prevalent in some groups than in others-for example, schizophrenia for migrants (Jones and Fernyhough 2007), or depression for women (Kessler 2003) - and which factors may act as protective at the group level (Kirkbride et al. 2007). 
more frequently and intensely, thus crossing the threshold from ARMS to clinical psychosis. I characterize these situations as ones in which the threshold to pathology is crossed by overflow. However, someone may also experience a sudden or progressive weakening of protective resources. This would be the case of a person who struggles with depressive symptoms while finding social and emotional support in a significant other. If this source of protection disappears-for example, if the other person dies or moves away-the transition to clinical relevance becomes more likely. In these situations, I talk about crossing the threshold by collapse.

As I clarify in the next section, the distinction between different paths hinges on the key notions of risk and protection. Psychologists working within the appraisal paradigm (Lazarus 1991; Lazarus and Folkman 1984) have characterized vulnerability in terms of resources mobilized in response to challenges. In this view, stress-inducing factors are those responsible for increasing vulnerability while stress-reducing factors usually contribute to diminishing it. Drawing on this idea, I characterize risk and protection as indicators that clinicians observe to assess how close an individual is to crossing a clinically relevant threshold. In this sense, indicators of risk and protection would work similarly to red flags or green lights. Taking them into account allows clinicians to home in on aspects of the person's situation that increase or decrease vulnerability, with the goals of making case formulation more individualized, monitoring the patient's trajectory more effectively, and intervening in the relevant factors whenever possible. In sections 3 and 4 I illustrate how contributing factors and paths interact in complex ways through a discussion of some significant cases. In section 3 I take a closer look at risk indicators (that is, increase in severity), while in section $4 \mathrm{I}$ focus on the notion of decreasing protection (that is, weakening of defenses).

\section{From Vulnerability to Clinical Relevance: Overflow}

When discussing the notion of clinical relevance, Janet B. Williams and Robert L. Spitzer (1982) talk about a group of conditions that can be considered "pathological," but the clinical manifestations are so mild that clinical attention is not indicated. In these cases an individual may be seen as vulnerable because they exhibit an attenuated version of a clinically relevant condition. For example, someone may be experiencing sadness, loss of motivation, or black-and-white thinking, without thereby crossing the diagnostic threshold for clinical depression. In these cases, the transition to clinical relevance occurs when some indicators of risk increase to a problematic degree, and we witness an increase in severity along relevant dimensions such as duration, frequency, urgency, or intensity. This implies that some phenomena are experienced and classified as unproblematic when they present themselves at a sufficiently low degree of frequency, intensity, and so on, whereas a quantitative increase in severity marks the difference between vulnerable and clinically relevant conditions.

A few examples may be helpful. As mentioned earlier, a person who is very sad and a clinically depressed patient may report qualitatively similar experiences. Sadness often includes a significant loss of pleasure or motivation in everyday activities and may be accompanied by physiological symptoms such as a change in appetite or sleep patterns. Both sad and depressed individuals show little interest in activities that others (or their former selves) regard as pleasurable; they both suffer from low mood and fatigue in carrying out everyday tasks, and so on (Horwitz and Wakefield 2007). However, intense sadness can be seen as a normal and even adaptive response to life stressors-for example, the loss of a valued job, divorce, or loss of status. The issue of reliably distinguishing 
between depression and normal sadness is notoriously thorny, to the point that past editions of the Diagnostic and Statistical Manual of Mental Disorders (DSM) included a number of exclusion clauses to circumvent this issue. ${ }^{6}$ Notably, there are cases in which the two conditions appear remarkably similar: they may both persist over long periods of time; they may both exhibit high intensity and absorb a great part of the person's physical and mental resources, and so on.

Similarly, the experience of hearing voices-AVH-has been found to be surprisingly frequent in the general population (Johns et al. 2014), despite being one of the hallmarks of schizophrenia. Over the past few decades, several studies have reported the existence of hallucinatory experiences-both visual and verbal-in samples of healthy college students (see Johns and Van Os 2001 for a review). For instance, 71\% of the subjects interviewed by Thomas B. Posey and Mary E. Losch (1983) reported some experience of at least brief, occasional hallucinated voices during periods of wakefulness ( $\mathrm{n}=375$ college students). Other studies highlight the relative frequency of hallucinatory experiences in healthy individuals: for instance, Terry R. Barrett and Jane B. Etheridge (1992) found that between 30 and $40 \%$ of their sample ( $n=586$ college students) reported AVH experiences, and almost half of these indicated a monthly occurrence. Notably, the phenomenology reported by clinical and nonclinical groups appears to be quite similar in terms of features such as localization (that is, internal or external), loudness, number of voices, and personification-that is, attribution of the voice to a real person or entity (Johns et al. 2014).

Another interesting example concerns autistic spectrum conditions (ASCs). In their comprehensive review, Ed Sucksmith, Ilona Roth, and Rosa Anna Hoekstra (2011) discuss a number of studies investigating the so-called broader autism phenotype (BAP). BAP may be characterized as a collection of subthreshold traits frequently found in the relatives of people diagnosed with autism, and especially in younger siblings (Merin et al. 2007). These milder manifestations of the condition usually consist of a wide range of features, such as delayed language development (Constantino et al. 2006) and social difficultiesfor example, atypical gaze shift and reduced initiation of joint attention (Presmanes et al. 2007). Although a number of studies report mixed results, especially in the domain of executive functions, theory of mind, and repetitive behavior, there is reason to believe that younger siblings of autistic children might constitute a vulnerable group (Cassel et al. 2007). BAP can thus be conceptualized as a set of continuous and quantitative traits, with some subgroups exhibiting a higher degree of risk. ${ }^{7}$

In these cases, and other similar ones, we may describe the transition from vulnerable to clinically relevant manifestations in terms of increase of severity along some relevant dimensions. Vulnerable manifestations represent attenuated versions of the clinically relevant conditions, in which a clinician would witness a similar clinical presentation at a lower degree of severity. What are some of the relevant dimensions at play in such a

\footnotetext{
${ }^{6}$ For instance, up to the DSM-IV the definition of "Major Depression" acknowledged that people who recently experienced the loss of a loved one were to be exempted from diagnosis even upon meeting all the criteria (the so-called Bereavement Clause, then removed from the DSM-5)-see Zachar, First, and Kendler (2017) for discussion.

7 It is worth noting that the use of notions such as "vulnerable" or "at risk" when it comes to autism spectrum conditions is potentially problematic, given that autism is generally understood as a neurodevelopmental condition (something one is born with). What is usually meant in the literature on BAP is that family members may exhibit subthreshold traits that are not severe enough to warrant diagnosis, but might require increased clinical attention, as well as some form of intervention (for example, accommodations in educational settings). Sucksmith, Roth and Hoekstra $(2011,261)$ also suggest that some BAP individuals may be diagnosed with autism later in life and thus exhibit a different, arguably less severe, developmental trajectory.
} 
transition? An important one is undoubtedly duration, which indicates how long a symptom, thought, or feeling extends over time. In terms of the sadness/depression examples above, having the blues for a couple of days differs from experiencing a comparable degree of sadness for months in a row. Another relevant dimension is frequency, indicating the number of times a symptom or experience presents itself to consciousness. For example, hearing voices a few times a month differs from having the same experience hundreds of times a day. In fact, some of the core differences between clinical and nonclinical voice hearers lie in the frequency and duration of AVH experiences (Johns et al. 2014).

Elements such as intensity and urgency also play a key role: while the former may be characterized in terms of strength or depth, the latter indicates how soon a problem needs to be dealt with, a thought or feeling acted upon, and so on. The notion of intensity is notoriously hard to pin down: a psychological manifestation may be regarded as intense by virtue of its strength, measured by the number of resources the subject has to mobilize in order to cope with it (Lazarus and Folkman 1984). In this sense, for instance, a thought qualifies as intense if dealing with it requires an exertion of effort that leaves a person unable to carry out any other task. This can be clearly seen in the role played by routines and rituals in conditions such as autism or obsessive-compulsive disorder (OCD), where intensity might be so strong as to significantly interfere with daily life and functioning. Yet, a psychological phenomenon may qualify as intense also by virtue of its depth-for example, by causing someone to radically revise her view on things. In this sense, a delusional idea may be intense because it transforms a person's relationship with the environment and gives rise to new interpretations of events and situations. Finally, scope should also be monitored closely because it concerns the number aspects of a situation that the individual regards as relevant. For example, it might be easier to control AVH symptoms when they are restricted to a specific domain (for example, one's job performance) and it might become progressively harder as they apply to more objects, people, or events. In the case of sadness and depression, it also makes sense to think that in most cases these two experiences would differ in terms of depth and scope. While depression casts a gloomy light on the person's overall existence, sadness tends to be more localized, and connected with a specific trauma or negative life event.

A brief discussion of these dimensions illustrates how in some cases the transition from vulnerability to pathology happens by overflow-through an increase in severity. To sum up: in a clinically relevant manifestation, we expect the relevant symptoms or experiences to last longer (duration), to be triggered more easily (frequency), to take up more time and resources to the point of interfering with everyday functioning (intensity), and to be more pervasive or all encompassing (urgency and scope). A vulnerable individual would score lower along most-if not all-of these dimensions, thereby exhibiting an attenuated form of a clinically relevant condition.

A final metaphor may be helpful to grasp this point at a more intuitive level. A vessel struggles to stay above water during a storm, while fighting against a number of dangers that may cause the water level inside the ship to increase. For the crew, it is crucial to assess how long the storm will last (duration), how likely it is for other storms to occur (frequency), and how strong this event will be in comparison to others (intensity). It is also important to assess how soon the storm will start damaging the ship's vital components (urgency), and how much effort and how many resources will be needed to repair it after the threat has ended (scope). Crucially, the breaking point will be reached when one or more of these factors cause the water level to increase to the point of overflow. 
Yet, sticking to the metaphor, the level of threat posed by the storm also importantly depends on the overall conditions of the vessel. If the lifeboats were lost during the last expedition, even a mild storm may qualify as dangerous. Conversely, the ship may have been recently repaired and prove more resistant in the face of adversities. This tells us that-besides indicators of risk-a thorough assessment of the vessel's condition has to include indicators of protection. In the next section I expand on this notion, and on the key role it plays in assessing vulnerability and clinical relevance.

\section{From Vulnerability to Clinical Relevance: Collapse}

In some cases an individual may be seen as vulnerable because she is experiencing a harmful psychological condition which-despite its severity-fails to qualify as clinically relevant due to the presence of one or more protective resources. For example, a person may suffer from a number of obsessions while still failing to count as pathological because she is able to detach herself from intrusive thoughts through humor. In these cases the transition to pathology may occur whenever the relevant protective resources become insufficient or weaken significantly. For instance, the person who is usually able to reframe her symptoms through humor might go through a particularly difficult time during which she lacks the energy to do so. If this happens, her obsessions may spiral out of control and significantly impair her functioning without increasing in severity (without becoming more frequent, intense, and so on). This path from vulnerability to clinical relevance involves a weakening of protection along relevant dimensions, such as control, humor, discharge, and physical or mental strength. In these cases the threshold to a clinically relevant condition is crossed because the person gradually or suddenly loses resources that were playing a key counterbalancing role in coping with a harmful situation. Without these protections in place, the person is thus more likely to exhibit a clinically relevant condition.

Some examples may be helpful to illustrate the point. Think about someone who scores high on the neuroticism scale, a collection of traits usually described as the tendency to experience anxiety, moodiness, worry, or frustration (Goldberg 1990). Paradigmatic examples are the so-called Woody Allen cases-people who take neuroticism as a defining feature of their personality (Killmister 2015). Woody Allen cases are interesting for our purposes because some of them exhibit quite a severe profile in terms of the risk indicators discussed in section 3. Take Allan, the protagonist of Allen's movie Play It Again Sam (1972): among many other idiosyncrasies, Allan has an obsession with Humphrey Bogart, who vividly shows up in verbal and visual hallucinatory experiences to coach him on how to deal with romantic matters. Despite the seemingly similar profile, we may draw some significant distinctions between Allan and a person who is clinically obsessed or psychotic. As mentioned earlier, some individuals-neurotic ones in particular-exhibit a striking tendency toward sarcasm and self-irony that may act as a buffer against developing clinically relevant manifestations. In other words, being able to recruit humor to laugh about their own idiosyncrasies and obsessions may provide them with the resources to exercise control, as well as to promote detachment from stressful situations.

Another interesting case concerns the group of phenomena known as postbereavement hallucinatory experiences (PBHEs). Although their nature remains elusive, these phenomena are usually taken to encompass a range of disturbances of perception and thought processes, such as hallucinations, pseudo-hallucinations, illusions, and felt presences (Castelnovo et al. 2015). PBHEs are often experienced by family members or 
close friends during-but also, significantly, after-the mourning period. In a pioneering study on the psychology of grief, Colin Murray Parkes (1972) distinguishes between the phenomenon of felt presence and full-blown hallucinations: the former experience is characterized by feeling the presence of the dead person without directly perceiving him or her (for example, "I still feel that he is around"), while the latter includes a strong perceptual component (for example, "I hear him and see him"). In the first quantitative study conducted on PBHEs, W. Dewi Rees (1971, 61-62) interviewed a group of Welsh widows who describe these experiences as being particularly frequent (from "variable times throughout the day" to "being continually hallucinated") and long lasting (spanning the first five to ten years of widowhood). In terms of risk indicators, it might be difficult to distinguish PBHEs from full-blown hallucinatory experiences. In fact, PBHEs may score even higher on the duration and frequency dimensions, considering that the process of bereavement tends to last several years as opposed to psychotic episodes that often resolve in a few days or weeks. While the degree of intensity is probably very high in both situations, there is one crucial difference: patients affected by hallucinations tend to feel persecuted or haunted by these experiences, whereas a great majority of the subjects interviewed by Rees present PBHEs in a positive light $(1971,40)$. Such a difference in valence indicates that people experiencing PBHEs probably benefit from the action of several protective resources. For instance, some reports indicate that these individuals are able to maintain a certain degree of control over the situation, by telling themselves that they are just imagining it-"I hear him and see him although I know it's only imagination" (Parkes 1972, 61-62)-or by being able to regard these experiences as private and as something that can be integrated in their everyday life without disrupting it (Parkes 1972, 41). In this sense, PBHEs might work as coping strategies themselves, as they help to fulfill a frustrated desire (for example, to see the loved one again) and function as ways of processing stressful experiences (for example, by talking them through with the deceased spouse).

In the cases discussed above, as well as in similar others, we may describe the transition from vulnerable to clinically relevant manifestations in terms of weakening of protection along relevant dimensions. Vulnerable individuals would thus exhibit profiles very similar to clinically relevant ones in terms of risk indicators (duration frequency, intensity, and so on), with protective resources playing a counterbalancing role. What do these protective resources look like? One key resource would surely be a person's ability to control a challenging situation by employing successful coping strategies. For example, one may attempt to avoid situations that would trigger obsessive thoughts, or direct attention elsewhere to change one's perspective on the issue-for example, by framing PBHEs as imaginative rather than perceptual experiences. ${ }^{8}$ Another important resource would be the amount of strength that an individual has at their disposal to handle the relevant challenges. Notably, the notion of strength consists of physical resources-such as getting enough sleep or food-as well as psychological ones, such as the ability to focus, solve problems, and regulate affect. Generally speaking, a sufficient degree of physical and psychical strength protects the subject from feeling overwhelmed by external or internal demands. In this sense, it is unsurprising that phenomena such as psychotic outbreaks often occur during particularly stressful times (for example, being appointed for a prestigious but demanding job, or going up for tenure-see Schreber 1903 and Saks 2007

\footnotetext{
${ }^{8}$ Here I draw again on appraisal theories of emotion to characterize control as the ability to employ one or more coping strategies to deal with stressors. See Gross (2002) for a more comprehensive classification of such strategies.
} 
for first-person accounts). Similarly, depressive episodes are usually characterized by a state of energy depletion where carrying out small, everyday tasks becomes impossible (see Plath 1963 and Styron 1991 for some relevant examples). Various forms of discharge may also act as protective resources because they allow individuals to release mental or emotional tension-for example, by talking to a friend, or writing in one's diary-as well as physical tension (for example, through physical activity, or crying). Although the idea that discharge may act in a protective way is quite old-see the notion of Entladung in Freud (1905), it has been more recently corroborated by evidence on the value of movement for mental health (see Craft and Perna 2004 on clinical depression), as well as on the effectiveness of talk therapy (Shedler 2010).

Finally, humor deserves a special place among protective resources because of its potential to act as a powerful tool to keep distressing thoughts and emotions at bay. This may be accomplished in a variety of ways. At times humor helps to promote detachment from dreadful or anxiety-inducing events-for example, through pre-surgery or pre-exam jokes (see Hardy 2020)-or achieves some form of release by voicing thoughts that are repressed or unacceptable (for example, politically incorrect jokes). In other circumstances-as mentioned earlier in the case of neuroticism-humor engenders a paradoxical effect. For example, someone who magnifies a negative personality trait or systematically draws attention to it through self-irony or self-deprecation ends up appearing more in control with respect to their situation. Exercising irony on one's own character flaws indicates some degree of self-reflection and ability to put things in perspective: Woody Allen's characters, for instance, appear painfully aware of the gap between them and others (for example, "Why can't I develop that attitude? Why is it always so complicated? Why didn't I see it coming?”). Assessing such a degree of control and self-reflection afforded by humor is particularly important when it comes to identifying clinically relevant situations. While neurotic individuals appear to actively engage-and even negotiate-with their symptoms (by joking about them, or even flaunting them as personality-defining features), clinically obsessed patients often lack such possibilities and end up adopting a passive attitude toward their thoughts and actions (by treating them as orders to be obeyed, or as inescapable failures).

Another metaphor might help us to grasp this alternative path from vulnerability to clinical relevance. Think about a fortress whose inhabitants attempt to defend themselves from a siege. As the attack unfolds, there are a number of countermeasures that the citizens can take to protect themselves. First, it is important to understand the enemy's strategy and to make decisions about how to better react (control). Assuming that the threat cannot be ignored, the inhabitants have to establish what can be done about it: some protective measures would involve rendering the fortress more resistant against the attack, while others would focus on preserving human and material resources for the longest possible time. For example, soldiers may arrange a watch system to ensure that everyone gets a minimum amount of sleep, or they may regulate the distribution of food and water (strength). In order to release the stress caused by the threatening situation, the inhabitants might also engage in activities directed towards the release of tension-telling jokes about the siege or getting drunk. If the situation became serious enough, the defenders would become progressively weaker and have fewer resources at their disposal. Some of the protective measures would then cease to be effective (joking about the siege is no longer fun), and others become plainly unavailable (for example, if the city runs out of wine). On the brink of disaster, the inhabitants may decide to keep fighting despite lacking the resources to sustain the attack any longer, thereby causing the fortress to collapse. 
Notably-in contrast to the vessel metaphor-the breaking point here is reached when the defenses put in place wear out; that is, through a weakening of protection.

\section{Clarifications and Concluding Remarks}

In this article I set out to make the notion of psychiatric vulnerability more precise by distinguishing different ways of being at risk of developing a clinically relevant condition. On the one hand, a person may be more vulnerable than others due to different contributing factors. A fine-grained assessment of such factors may contribute to determining the position that an individual (or group) occupies along the continuum between clinical and nonclinical. In this sense, someone with a tendency toward rumination and a traumatic past may be classified as being more vulnerable to developing clinical depression compared with someone who does not exhibit these features. On the other hand, individuals (or groups) placed along this continuum may develop a clinically relevant manifestation through different paths. In sections 3 and 4 I explore two of them, showing that-given an existing degree of vulnerability-the threshold to clinical relevance can be crossed through an increase in severity (overflow) or through a decrease in protection (collapse).

One important clarification is in order. The distinctions I propose should be regarded as helpful ways to illustrate how we should think about vulnerability in psychiatric practice. However, given the complexity discussed in this article, these distinctions should not be applied too rigidly when it comes to assessing individual cases. For instance, overflow and collapse should be interpreted as situations in which the threshold to pathology is crossed mostly via an increase in severity or via a weakening in protection. Individual cases are obviously going to be more complex, as often both aspects play an important role and deeply influence each other. An increase in severity may render some of the protective resources ineffective or unavailable. For instance, if an obsessive thought increases in frequency, intensity, and urgency, exercising control over it becomes more difficult and detachment strategies (such as humor) may prove impossible to implement. By contrast, vulnerable situations where severity increases may be mitigated by the recruitment of protective resources: a person on the verge of clinical depression may adopt a number of coping strategies, including self-reassuring beliefs, cognitive changes, or selfirony. On the other hand, a weakening of protection may contribute to an increase in severity. For instance, a recovering alcoholic who does not manage to avoid her circle of drinking friends would probably end up thinking about the next drink more frequently and experiencing the thought as more urgent.

The interaction between risk and protection may therefore prove quite hard to disentangle, with contextual contingencies playing a key role in individual cases. Freud expresses a similar idea of vulnerability through the metaphor of a sliding scale, where multiple factors converge to constitute the etiology of an individual patient: "Generally the two factors [constitutional and accidental] work together and only at either end of the series can we speak of a simple motivation" $(1939,119)$.

The interaction between contributing factors and paths, as well as between risk and protection, is bound to be extremely complex. Although distinguishing different aspects of psychiatric vulnerability enhances conceptual clarity, it is often unfeasible to neatly identify contributing factors and paths in a clinical setting. Arguably, in most situations the transition to clinical relevance would therefore occur via a complex interplay between risk, protection, and the intersection between individual and environmental vulnerability. 
Having a clearer idea of the individual's current degree of vulnerability, and of the possible ways in which they could cross the threshold to a clinically relevant condition, may support clinicians in the diagnostic and therapeutic process. For instance, psychiatrists may employ these distinctions to determine where a patient lies along the course of an illness (McGorry et al. 2010), or to better understand subthreshold conditions and prodromal phases of disorders (Fusar-Poli et al. 2016). Yet, such a focus on vulnerability should always be complemented by an awareness of the possibility of "false positives," especially in situations where this might lead to unnecessary and potentially invasive treatment. This point is particularly important in screening programs for psychosis, where only a minority of individuals included in prodromal services have been found to actually transition to a clinically relevant condition (Ajnakina et al. 2017) for reasons that are still poorly understood (Riecher-Rössler and Studerus 2017). As Derek Bolton (2008) rightfully emphasizes, it is crucial to balance the need to treat distress or disability with the potential drawbacks that might follow a referral to mental health services and a subsequent diagnosis (such as stigma and financial costs).

Given the scarce philosophical literature on vulnerability in psychiatry, several important issues remain that cannot be addressed satisfactorily in this article. Key among them, as already mentioned, is the role of environmental factors-in particular social elements (experiences of discrimination, defeat, and so on)-in determining individual and population degrees of vulnerability. Indeed, it is particularly important to acknowledge that the notions of risk and protection are not limited to individual-level strategies and may be environmental or structural in nature. For instance, someone may be struggling with severe depressive symptoms while finding social and emotional support in a significant other, whereas someone else may be more exposed to negative life experiences due to their gender, ethnic background, socioeconomic status, and so on. I plan on exploring these aspects in a future project devoted to the impact of social adversity on psychiatric vulnerability. An ideal starting point would be the body of research on group-level exclusion and discrimination (Cantor-Graae and Selten 2005; Bourque, Van der Ven, and Malla 2011; Berg et al. 2011), and the epidemiological data on group prevalence for some psychiatric conditions-such as schizophrenia for migrants (Jones and Fernyhough 2007) and depression for women (Kessler 2003).

A related issue concerns the notion of resilience and its connection to adversity. While this article illustrates different ways in which people may develop clinically relevant conditions, an equally important challenge for psychiatry would be to investigate the factors that make people resistant to mental disorders even when confronted with considerable pressure and adversity. This issue was repeatedly raised by Freud (1924, 1926), who observed that people who share a similar psychological makeup and go through similar life experiences might end up developing very different psychopathological trajectories. Again, the connection between resilience and adversity is bound to be particularly complex. While there is evidence that exposure to environmental stressors may contribute to the development of forms of protective resistance (Rutter 1985; Bandura 1997), others have emphasized that such a repeated exposure may engender a sensitization effect, which produces progressively greater responses over time (Collip, Myin-Germeys, and Van Os 2008). "Why are some people not falling ill?" (Freud 1924, 152; emphasis added) might therefore represent an intriguing question to explore in future work on psychiatric vulnerability. 


\section{Acknowledgments}

I have worked on the ideas presented in this article over several years, and I have benefited from fruitful discussions and exchanges with many friends, colleagues, and mentors. Earlier versions of this material were discussed with Johannes Brandl, to whom credit is also due for the vessel and fortress metaphors, as well as with Peter Langland-Hassan, Heidi Maibom, Tom Polger, and the colleagues at the lunchtime work-in-progress seminar at the University of Cincinnati. Later versions were discussed at the 2019 Italian Association of Cognitive Sciences (AISC) meeting in Rome, and at Lindy Lab seminars at the University of the Basque Country (UPV/EHU). For muchneeded encouragement and helpful feedback throughout the process, I am grateful to Davide Serpico, Marco Viola, Agustín Vicente, Marta Jorba, Elena Castroviejo, Alexander Jeuk, Irene Binini, and Federica Bocchi. I am also thankful to the editors and two anonymous referees for Philosophy of Medicine, whose feedback and questions significantly contributed to improving this article.

My research is supported by Ministerio de Ciencia, Innovación y Universidades, postdoctoral fellowship FJC2018-036191-I; by Agencia Estatal de Investigación, grant number: PGC2018093464-B-Ioo; by Eusko Jaurlaritza (the Basque government), grant number: IT1396-19; and by Euskal Herriko Unibertsitatea (the University of the Basque Country-UPV/EHU), grant number: GIU18/221.

\section{Disclosure Statement}

No competing interests were reported by the author.

\section{References}

Ajnakina, Olesya, Anthony S. David, and Robin M. Murray. 2019. “'At Risk Mental State' Clinics for Psychosis-an Idea Whose Time Has Come-and Gone!” Psychological Medicine 49, no. 4: 529534. https://doi.org/10.1017/s0033291718003859.

Ajnakina, Olesya, Craig Morgan, Charlotte Gayer-Anderson, Sherifat Oduola, François Bourque, Sally Bramley, Jessica Williamson et al. 2017. "Only a Small Proportion of Patients with First Episode Psychosis Come via Prodromal Services: A Retrospective Survey of a Large UK Mental Health Programme." BMC Psychiatry 17, no. 1: 1-9. https://doi.org/10.1186/s12888-017-1468-y.

Allen, Woody, dir. 1972. Play It Again Sam. Los Angeles: Paramount Pictures.

Allsopp, Kate, John Read, Rhiannon Corcoran, and Peter Kinderman. 2019. "Heterogeneity in Psychiatric Diagnostic Classification.” Psychiatry Research 279: 15-22.

https://doi.org/10.1016/j.psychres.2019.07.005.

Bandura, Albert. 1997. Self-Efficacy: The Exercise of Control. New York: W.H. Freeman \& Company.

Barrett, Terry R. and Jane B. Etheridge. 1992. "Verbal Hallucinations in Normals, I: People Who Hear 'Voices'." Applied Cognitive Psychology 6, no. 5: 379-387.

https://doi.org/10.1002/acp.2350060503.

Berg, Akiah O., Ingrid Melle, Jan Ivar Rossberg, Kristin Lie Romm, Sara Larsson, Trine V. Lagerberg, Ole A. Andreassen, and Edvard Hauff. 2011. "Perceived Discrimination Is Associated with Severity of Positive and Depression-Anxiety Symptoms in Immigrants with Psychosis: A Cross-Sectional Study." BioMed Central Psychiatry 11: 77-86. https://doi.org/10.1186/1471-244X11-77.

Bluhm, Robyn. 2012. "Vulnerability, Health, and Illness." International Journal of Feminist Approaches to Bioethics 5, no. 2: 147-161. https://doi.org/10.2979/intjfemappbio.5.2.147. 
Boldt, Joachim. 2019. "The Concept of Vulnerability in Medical Ethics and Philosophy." Philosophy, Ethics, and Humanities in Medicine 14, no. 1: 1-8. https://doi.org/10.1186/s13010019-0075-6.

Bolton, Derek. 2008. What Is Mental Disorder? An Essay in Philosophy, Science, and Values. Oxford: Oxford University Press.

Bourque, François, Elsje van der Ven, and Ashok Malla. 2011. "A Meta-analysis of the Risk for Psychotic Disorders among First- and Second-Generation Immigrants." Psychological Medicine 41, no. 5: 897-910. https://doi.org/10.1017/s0033291710001406.

Broome, Matthew R., James B. Woolley, Louise C. Johns, Lucia R. Valmaggia, Paul Tabraham, Rafael Gafoor, Elvira Bramon, and Philip K. McGuire. 2005. "Outreach and Support in South London (OASIS): Implementation of a Clinical Service for Prodromal Psychosis and the At-Risk Mental State." European Psychiatry 20, no. 5-6: 372-378.

https://doi.org/10.1016/j.eurpsy.2005.03.001.

Cantor-Graae, Elizabeth and Jean-Paul Selten. 2005. "Schizophrenia and Migration: A Metaanalysis and Review.” American Journal of Psychiatry 162, no. 1: 12-24.

https://doi.org/10.1176/appi.ajp.162.1.12.

Cassel, Tricia D., Daniel S. Messinger, Lisa V. Ibanez, John D. Haltigan, Susan I. Acosta, and Albert C. Buchman. 2007. "Early Social and Emotional Communication in the Infant Siblings of Children with Autism Spectrum Disorders: An Examination of the Broad Phenotype." Journal of Autism and Developmental Disorders 37, no. 1: 122-132. https://doi.org/10.1007/s10803-006-0337-1.

Castelnovo, Anna, Simone Cavallotti, Orsola Gambini, and Armando D’Agostino. 2015. "Postbereavement Hallucinatory Experiences: A Critical Overview of Population and Clinical Studies." Journal of Affective Disorders 186: 266-274. https://doi.org/10.1016/j.jad.2015.07.032.

Clark, Beth and Nina Preto. 2018. "Exploring the Concept of Vulnerability in Health Care." CMAJ 190, no. 11: E308-E309. https://doi.org/10.1503/cmaj.180242.

Collip, Dina, Inez Myin-Germeys, and Jim van Os. 2008. “Does the Concept of 'Sensitization' Provide a Plausible Mechanism for the Putative Link between the Environment and Schizophrenia?" Schizophrenia Bulletin 34, no. 2: 220-225. https://doi.org/10.1093/schbul/sbm163.

Constantino, John N., Clara Lajonchere, Marin Lutz, Teddi Gray, Anna Abbacchi, Kathleen McKenna, Deepti Singh, and Richard D. Todd. 2006. "Autistic Social Impairment in the Siblings of Children with Pervasive Developmental Disorders." American Journal of Psychiatry 163, no. 2: 294-296. https://doi.org/10.1176/appi.ajp.163.2.294.

Craft, Lynette L. and Frank M. Perna. 2004. "The Benefits of Exercise for the Clinically Depressed." Primary Care Companion to the Journal of Clinical Psychiatry 6, no. 3: 104-111.

https://dx.doi.org/10.4088\%2Fpcc.vo6no301.

Drożdżowicz, Anna. 2021. "Epistemic Injustice in Psychiatric Practice: Epistemic Duties and the Phenomenological Approach." Journal of Medical Ethics. https://doi.org/10.1136/medethics2020-106679.

Fairbrother, Nichole and Sheila R. Woody. 2008. "New Mothers' Thoughts of Harm Related to the Newborn." Archives of Women's Mental Health 11, no. 3: 221-229. https://doi.org/10.1007/s00737-008-0016-7.

Fearon, Paul, James B. Kirkbride, Craig Morgan, Paola Dazzan, Kevin Morgan, Tuhina Lloyd, Gerard Hutchinson et al. 2006. "Incidence of Schizophrenia and Other Psychoses in Ethnic 
Minority Groups: Results from the MRC AESOP Study." Psychological Medicine 36, no. 11: 15411550. https://doi.org/10.1017/s0033291706008774.

Foucault, Michel. 1961. Madness and Civilization: A History of Insanity in the Age of Reason. New York: Vintage.

Freud, Sigmund. 1905. "Jokes and Their Relation to the Unconscious." In The Standard Edition of the Complete Psychological Works of Sigmund Freud, Vol. 8, edited and translated by James Strachey, 237-245. London: Hogarth.

---. 1913. "The Disposition to Obsessional Neurosis." In The Standard Edition of the Complete Psychological Works of Sigmund Freud, Vol. 12, edited and translated by James Strachey, 313327. London: Hogarth.

- - . 1924. "Neurosis and Psychosis." In The Standard Edition of the Complete Psychological Works of Sigmund Freud, Vol. 19, edited and translated by James Strachey, 149-156. London: Hogarth.

--_. 1926. "Inhibitions, Symptoms and Anxiety. In The Standard Edition of the Complete Psychological Works of Sigmund Freud, Vol. 20, edited and translated by James Strachey, 77-178. New York: W.W. Norton \& Company.

---. 1939. "Moses and Monotheism." In The Standard Edition of the Complete Psychological Works of Sigmund Freud, Vol. 23, edited and translated by James Strachey, 1-38. London: Hogarth.

Fulford, K.W.M. 1989. Moral Theory and Medical Practice. Cambridge: Cambridge University Press.

Fusar-Poli, Paolo, Stefan Borgwardt, Andreas Bechdolf, Jean Addington, Anita Riecher-Rössler, Frauke Schultze-Lutter, Matcheri Keshavan et al. 2013. "The Psychosis High-Risk State: A Comprehensive State-of-the-Art Review.” JAMA Psychiatry 70, no. 1: 107-120. https://doi.org/10.1001/jamapsychiatry.2013.269.

Fusar-Poli, P., G. Rutigliano, D. Stahl, C. Davies, A. de Micheli, V. Ramella-Cravaro, I. Bonoldi, and P. McGuire. 2016. "Long-Term Validity of the At Risk Mental State (ARMS) for Predicting Psychotic and Non-psychotic Mental Disorders." European Psychiatry 42: 49-54. https://doi.org/10.1016/j.eurpsy.2016.11.010.

Gilson, Erinn. 2011. "Vulnerability, Ignorance, and Oppression." Hypatia 26, no. 2: 308-332. https://doi.org/10.1111/j.1527-2001.2010.01158.x.

Goldberg, L.R. 1990. “An Alternative Description of Personality: The Big-Five Factor Structure.” Journal of Personality and Social Psychology 59, no. 6: 1216-1229.

https://doi.org/10.1037//0022-3514.59.6.1216.

Gross, James J. 2002. "Emotion Regulation: Affective, Cognitive, and Social Consequences.” Psychophysiology 39, no. 3: 281-291. https://doi.org/10.1017/s0048577201393198.

Hardy, Carter. 2020. "Humor and Sympathy in Medical Practice." Medicine, Health Care and Philosophy 23, no. 2: 179-190. https://dx.doi.org/10.1007\%2Fs11019-019-09928-0.

Horwitz, Alan V. and Jerome C. Wakefield. 2007. The Loss of Sadness: How Psychiatry Transformed Normal Sorrow into Depressive Disorder. New York: Oxford University Press.

Johns, Louise C., Kristiina Kompus, Melissa Connell, Clara Humpston, Tania M. Lincoln, Eleanor Longden, Antonio Preti et al. 2014. "Auditory Verbal Hallucinations in Persons with and without a 
Need for Care." Schizophrenia Bulletin 40, suppl. 4: S255-S264.

https://doi.org/10.1093/schbul/sbu005.

Johns, Louise C. and Jim van Os. 2001. "The Continuity of Psychotic Experiences in The General Population." Clinical Psychology Review 21, no. 8: 1125-1141. https://doi.org/10.1016/So2727358(01)00103-9.

Jones, Simon R. and Charles Fernyhough. 2007. "A New Look at the Neural Diathesis-Stress Model of Schizophrenia: The Primacy of Social-Evaluative and Uncontrollable Situations." Schizophrenia Bulletin 33, no. 5: 1171-1177. https://doi.org/10.1093/schbul/sblo58.

Kessler, Ronald C. 2003. "Epidemiology of Women and Depression.” Journal of Affective Disorders 74, no. 1: 5-13. https://doi.org/10.1016/s0165-0327(02)00426-3.

Killmister, Suzy. 2015. “The Woody Allen Puzzle: How ‘Authentic Alienation' Complicates Autonomy." Noûs 49, no. 4: 729-747. https://doi.org/10.1111/nous.12069.

Kingma, Elselijn. 2017. “Disease as Scientific and as Value-Laden Concept.” In Handbook of the Philosophy of Medicine, edited by T. Schramme and S. Edwards, 45-63. Dordrecht: Springer. https://doi.org/10.1007/978-94-017-8688-1 75.

Kirkbride, James B., Craig Morgan, Paul Fearon, Paola Dazzan, Robin M. Murray, and Peter B. Jones. 2007. "Neighbourhood-Level Effects on Psychoses: Re-examining the Role of Context." Psychological Medicine 37, no. 10: 1413-1425. http://dx.doi.org/10.1017/So033291707000499.

Kottow, Michael H. 2003. "The Vulnerable and the Susceptible." Bioethics 17, no. 5-6: 460-471. https://doi.org/10.1111/1467-8519.00361.

Lazarus, Richard S. 1991. "Cognition and Motivation in Emotion." American Psychologist 46, no. 4: 352-367. https://doi.org/10.1037//0003-066x.46.4.352.

Lazarus, Richard S. and Susan Folkman. 1984. Stress, Appraisal, and Coping. New York: Springer.

Lewis-Fernández, Roberto and Lawrence J. Kirmayer. 2019. "Cultural Concepts of Distress and Psychiatric Disorders: Understanding Symptom Experience and Expression in Context." Transcultural Psychiatry 56, no. 4: 786-803. https://doi.org/10.1177/1363461519861795.

Maj, Mario. 2011. "When Does Depression Become a Mental Disorder?” British Journal of Psychiatry 199, no. 2: 85-86. https://doi.org/10.1192/bjp.bp.110.089094.

McGorry, Patrick D., Ian B. Hickie, Alison R. Yung, Christos Pantelis, and Henry J. Jackson. 2006. "Clinical Staging of Psychiatric Disorders: A Heuristic Framework for Choosing Earlier, Safer and More Effective Interventions.” Australian \& New Zealand Journal of Psychiatry 40, no. 8: 616622. https://doi.org/10.1080/j.1440-1614.2006.01860.x.

McGorry, Patrick D., Barnaby Nelson, Sherilyn Goldstone, and Alison R. Yung. 2010. "Clinical Staging: A Heuristic and Practical Strategy for New Research and Better Health and Social Outcomes for Psychotic and Related Mood Disorders." Canadian Journal of Psychiatry 55, no. 8: 486-497. https://doi.org/10.1177/070674371005500803.

Merin, Noah, Gregory S. Young, Sally Ozonoff, and Sally J. Rogers. 2007. "Visual Fixation Patterns during Reciprocal Social Interaction Distinguish a Subgroup of 6-Month-Old Infants At-Risk for Autism from Comparison Infants." Journal of Autism and Developmental Disorders 37, no. 1: 108-121. https://doi.org/10.1007/s10803-006-0342-4.

Misra, Supriya, Bizu Gelaye, David R. Williams, Karestan C. Koenen, Christina P.C. Borba, Diego Quattrone, Marta di Forti et al. 2021. "Perceived Major Experiences of Discrimination, Ethnic 
Group, and Risk of Psychosis in a Six-Country Case-Control Study.” Psychological Medicine: 1-9. https://doi.org/10.1017/s0033291721000453.

Modinos, Gemma, P. Allen, Marianna Frascarelli, Stefania Tognin, Lucia Rita Valmaggia, LidaAlkisti Xenaki, Paul Keedwell et al. 2014. "Are We Really Mapping Psychosis Risk?

Neuroanatomical Signature of Affective Disorders in Subjects at Ultra High Risk." Psychological Medicine 44, no. 16: 1-11. http://dx.doi.org/10.1017/So033291714000865.

Murphy, Dominic. 2006. Psychiatry in the Scientific Image. Cambridge, MA: The MIT Press.

Murray, Robin M., Anthony S. David, and Olesya Ajnakina. 2021. "Prevention of Psychosis: Moving On from the At-Risk Mental State to Universal Primary Prevention." Psychological Medicine 51, no. 2: 223-227. https://doi.org/10.1017/s003329172000313x.

Nolen-Hoeksema, Susan, Blair E. Wisco, and Sonja Lyubomirsky. 2008. "Rethinking Rumination." Perspectives on Psychological Science 3, no. 5: 400-424. https://doi.org/10.1111/j.174.56924.2008.00088.x.

Nordenfelt, Lennart. 2007. "The Concepts of Health and Illness Revisited." Medicine, Health Care and Philosophy 10, no. 1: 5-10. https://doi.org/10.1007/s11019-006-9017-3.

Ormel, Johan and Jan Neeleman. 2001. "Towards a Dynamic Stress-Vulnerability Model of Depression." In Where Inner and Outer Worlds Meet: Psychosocial Research in the Tradition of George W. Brown, edited by Tirril Harris, 151-170. London: Routledge.

Parkes, Colin Murray. 1972. Bereavement: Studies of Grief in Adult Life. London: Tavistock Publications.

Plath, Sylvia. 1963. The Bell Jar. London: Faber \& Faber.

Posey, Thomas B. and Mary E. Losch. 1983. "Auditory Hallucinations of Hearing Voices in 375 Normal Subjects.” Imagination, Cognition and Personality 3, no. 2: 99-113. https://doi.org/10.2190\%2F74V5-HNXN-JEY5-DG7W.

Powell, Russell and Eric Scarffe. 2019. “Rethinking 'Disease': A Fresh Diagnosis and a New Philosophical Treatment.” Journal of Medical Ethics 45, no. 9: 579-588.

http://doi.org/10.1136/medethics-2019-105465.

Presmanes, Alison G., Tedra A. Walden, Wendy L. Stone, and Paul J. Yoder. 2007. "Effects of Different Attentional Cues on Responding to Joint Attention in Younger Siblings of Children with Autism Spectrum Disorders." Journal of Autism and Developmental Disorders 37, no. 1: 133-144. https://doi.org/10.1007/s10803-006-0338-0.

Rees, W. Dewi. 1971. "The Hallucinations of Widowhood." British Medical Journal 4, no. 5778: 3741. https://dx.doi.org/10.1136\%2Fbmj.4.5778.37.

Riecher-Rössler, Anna and Erich Studerus. 2017. "Prediction of Conversion to Psychosis in Individuals with an At-Risk Mental State: A Brief Update on Recent Developments." Current Opinion in Psychiatry 30, no. 3: 209-219. https://doi.org/10.1097/yc0.0000000000000320.

Rutter, Michael. 1985. "Resilience in the Face of Adversity: Protective Factors and Resistance to Psychiatric Disorder.” British Journal of Psychiatry 147, no. 6: 598-611. https://doi.org/10.1192/bjp.147.6.598.

Saks, Elyn R. 2007. The Center Cannot Hold: My Journey through Madness. London: Hachette Books. 
Schreber, Daniel Paul. 1903. Memoirs of My Nervous Illness. New York: New York Review of Books.

Serpico, Davide. 2020. "Beyond Quantitative and Qualitative Traits: Three Telling Cases in the Life Sciences.” Biology \& Philosophy 35, no. 3: 1-26. https://doi.org/10.1007/s10539-020-09750-6.

Shedler, Jonathan. 2010. "The Efficacy of Psychodynamic Psychotherapy." American Psychologist 65, no. 2: 98-109. https://doi.org/10.1037/a0018378.

Styron, William. 1991. Darkness Visible. New York: Random House.

Sucksmith, Ed, Ilona Roth, and Rosa Anna Hoekstra. 2011. "Autistic Traits below the Clinical Threshold: Re-examining the Broader Autism Phenotype in the 21st Century." Neuropsychology Review 21, no. 4: 360-389. https://doi.org/10.1007/s11065-011-9183-9.

Szasz, Thomas S. 1960. “The Myth of Mental Illness.” American Psychologist 15, no. 2: 113-118. https://doi/10.1037/ho046535.

Tate, Andrew James Miller. 2019. “Contributory Injustice in Psychiatry.” Journal of Medical Ethics 45, no. 2: 97-100. https://dx.doi.org/10.1136\%2Fmedethics-2018-104761.

Tavaglione, Nicolas, Angela K. Martin, Nathalie Mezger, Sophie Durieux-Paillard, Anne François, Yves Jackson, and Samia A. Hurst. 2015. "Fleshing Out Vulnerability." Bioethics 29, no. 2: 98-107. https://doi.org/10.1111/bioe.12065.

Travaglia, Joanne and Hamish Robertson. 2013. "Why Vulnerability Matters More Than Risk." https://www.academia.edu/5846341/Why vulnerability matters more than risk.

Turner, R. Jay and William R. Avison. 2003. "Status Variations in Stress Exposure: Implications for the Interpretation of Research on Race, Socioeconomic Status, and Gender." Journal of Health and Social Behavior 44, no. 4: 488-505.https://doi.org/10.2307/1519795.

Turner, R. Jay and Donald A. Lloyd. 1999. "The Stress Process and the Social Distribution of Depression." Journal of Health and Social Behavior 40, no. 4: 374-404.

https://doi.org/10.2307/2676332.

Van den Bergh, Omer, Jos Brosschot, Hugo Critchley, Julian F. Thayer, and Cristina Ottaviani. 2020. "Better Safe than Sorry: A Common Signature of General Vulnerability for Psychopathology." Perspectives on Psychological Science. https://doi.org/10.1177\%2F174569162095069o.

Van Os, Jim. 2013. "The Dynamics of Subthreshold Psychopathology: Implications for Diagnosis and Treatment." American Journal of Psychiatry 170, no. 7: 695-698.

https://doi.org/10.1176/appi.ajp.2013.13040474.

Van Os, J., R.J. Linscott, I. Myin-Germeys, P. Delespaul, and L.J.P.M. Krabbendam. 2009. “A Systematic Review and Meta-analysis of the Psychosis Continuum: Evidence for a Psychosis Proneness-Persistence-Impairment Model of Psychotic Disorder.” Psychological Medicine 39, no. 2: 179-195. https://doi.org/10.1017/s0033291708003814.

Veling, Wim, Ezra Susser, Jim van Os, Johan P. Mackenbach, Jean-Paul Selten, and Hans W. Hoek. 2008. "Ethnic Density of Neighborhoods and Incidence of Psychotic Disorders among Immigrants." American Journal of Psychiatry 165, no. 1: 66-73. https://doi.org/10.1176/appi.ajp.2007.07030423.

Vetter, Barbara. 2014. "Dispositions without Conditionals." Mind 123, no. 489: 129-156. http://dx.doi.org/10.1093/mind/fzuo32. 
Williams, J.B. and R.L. Spitzer. 1982. "Research Diagnostic Criteria and DSM-III: An Annotated Comparison." Archives of General Psychiatry 39, no. 11: 1283-1289.

https://doi.org/10.1001/archpsyc.1982.04290110039007.

World Health Organization. 1992. The ICD-10 Classification of Mental and Behavioural Disorders: Clinical Descriptions and Diagnostic Guidelines. Geneva: World Health Organization.

Zachar, Peter, Michael B. First, and Kenneth S. Kendler. 2017. "The Bereavement Exclusion Debate in the DSM-5: A History.” Clinical Psychological Science 5, no. 5: 890-906.

https://doi.org/10.1177\%2F2167702617711284.

Zubin, Joseph and Bonnie Spring. 1977. "Vulnerability: A New View of Schizophrenia." Journal of Abnormal Psychology 86, no. 2: 103-126. https://doi.org/10.1037//0021-843x.86.2.103. 